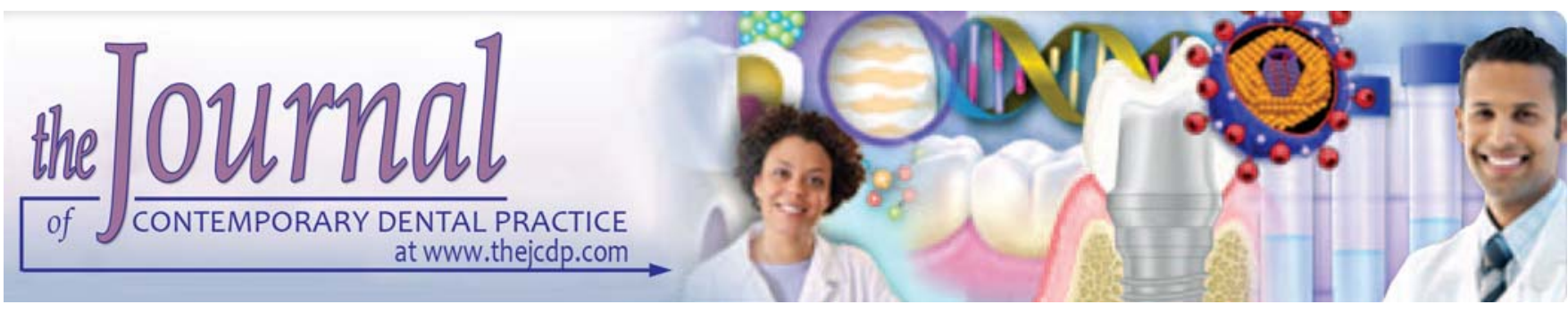

\title{
Maxillomandibular Plane Angle Bisector (MM) Adjunctive to Occlusal Plane to evaluate Anteroposterior Measurement of Dental Base
}

\author{
Chanamallappa R Ganiger, US Krishna Nayak, KU Cariappa, AR Yusuf Ahammed
}

\begin{abstract}
Aim: This study was undertaken to analyze the clinical usefulness of the maxillomandibular bisector, its reproducibility, its validity and its relationship to the functional occlusal plane, the bisecting occlusal plane and the nature of its cant.

Materials and methods: Thirty pretreatment lateral cephalograms, each of adolescents (above 18 years of age) and children (1012 years), seeking orthodontic treatment were randomly selected and the Wits technique of anteroposterior measurement was used to compare A-B values measured to the new plane with those measured to the functional occlusal plane (FOP) and to the traditional or bisecting occlusal plane (BOP).
\end{abstract}

Results: Present study showed that MM bisector plane is more reproducible and valid reference plane, than the FOP and BOP.

Conclusion: A new plane, geometrically derived from the dental base planes, has been tested as an occlusal plane substitute for the measurement of anteroposterior jaw relationships. It lies close to but at an angle and inferior to the traditional occlusal planes and is highly reproducible at all times.

Clinical significance: Maxillomandibular planes angle bisector may be a useful adjunct for the cephalometric assessment of sagittal relationship of the patient.

Keywords: Point A, point B, B isecting occlusal plane, Functional occlusal plane, Maxillomandibular planes angle.

How to cite this article: Ganiger CR, Nayak USK, Cariappa $K U$, Ahammed ARY. Maxillomandibular Plane Angle Bisector (MM) Adjunctive to Occlusal Plane to evaluate Anteroposterior Measurement of Dental Base. J Contemp Dent Pract 2012; 13(4):539-544.

Source of support: $\mathrm{Nil}$

Conflict of interest: None declared

\section{INTRODUCTION}

The goal of orthodontic treatment is to improve the patient's life by enhancing dentofacial esthetics and function. Diagnosis of an important part of orthodontic treatment and the greatest thrust in this direction evolved with advent of radiographic cephal ometry in 1931 by B roadbent. ${ }^{1} \mathrm{~A}$ Ithough many cephalometric analysis have been devised to determine the degree of anteroposterior skeletal discrepancies, effective treatment planning and assessment must be based on accurate measurement using stable and reproducible reference planes. Despite the popularity of the $A N B^{2}$ angle, Jacobson noted that this measurement does not always accurately relate the true anteroposterior relationship of the jaws.

$V$ ery few attempts have been made to develop an analysis of anteroposterior jaw relationship to reduce these problems. ${ }^{3}$ Jenkins and Harvold ${ }^{4,5}$ used the functional occlusal plane (FOP) as suitable reference plane for anteroposterior jaw disharmony assessment, J acobson ${ }^{2}$ suggested using the $W$ its appraisal to exclude the problems of a cranial base references which involves drawing perpendiculars form point $A$ and $B$ on the maxilla and mandible respectively, for the functional occlusal plane. How ever, there are several shortcomings to the Wits analysis, since the functional occlusal plane is difficult to identify and reproduce in missing teeth, malpositioned teeth, dental restorations, molar overlap and third molars or young patients with a pronounced curve of spee. The functional occlusal plane has also been shown to rotate in a random fashion during growth. ${ }^{5}$

\section{OBJECTIVE}

The purpose of this investigation was to evaluate the reliability and validity of anterioposterior skeletal measurement using the maxillomandibular angles bisector, FOP and BOP.

\section{MATERIALS AND METHODS}

Thirty pretreatment lateral cephal ograms each of adolescents (above 18 years of age) and children (10-12 years) seeking orthodontic treatment in D epartment of Orthodontics at A B Shetty M emorial Institute of Dental Sciences were randomly selected. 
Each film was hand traced by a single operator onto acetate tracing paper with $2 \mathrm{H}$ pencil locating the following anatomical landmarks (Fig. 1). Cross-examination was performed by the same examiner in order to eliminate examiner bias. The following planes were drawn SN, NA, NB, maxillary plane (A NS-PNS) mandibular plane (Go-M e) functional occlusal plane, bisecting occlusal plane and Enlow's vertical plane (SE-Ptm; Fig. 2). The maxillomandibular plane angle bisector was constructed and angular and linear measurements were performed.

\section{Cephalometric Measurements were Made using following Points and Planes}

Following points were constructed for the purpose of the study (Fig. 3).

Ao: Point A projected in perpendicular fashion onto the functional occlusal plane.

Bo: Point B projected in perpendicular fashion onto the functional occlusal plane.

Am: Point A projected in perpendicular fashion onto the MM bisector.

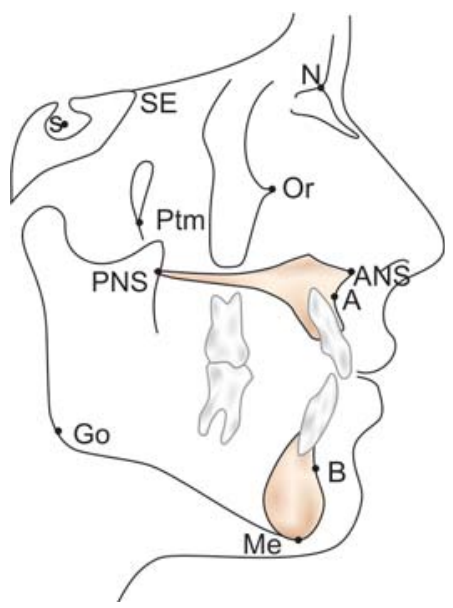

Fig. 1: Anatomical landmarks

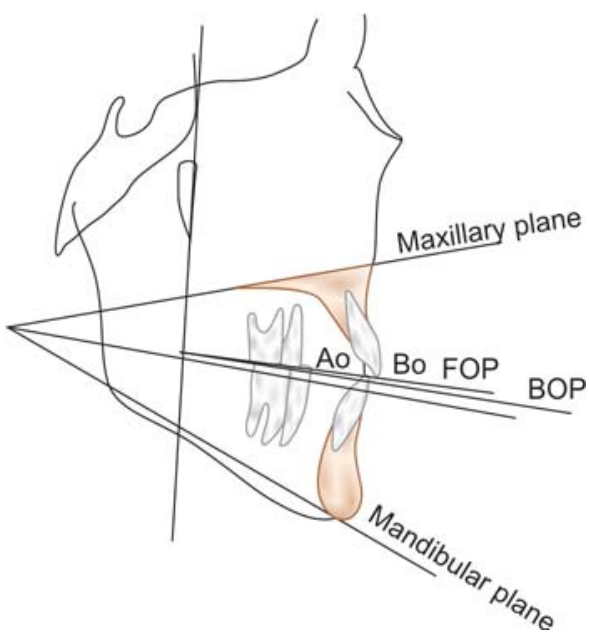

Fig. 2: Cephalometric planes used maxillary plane, mandibular plane, functional occlusal plane (FOP), bisecting occlusal plane (BOP), $A^{0}$ : point $A, B^{0}$ : point $B$.

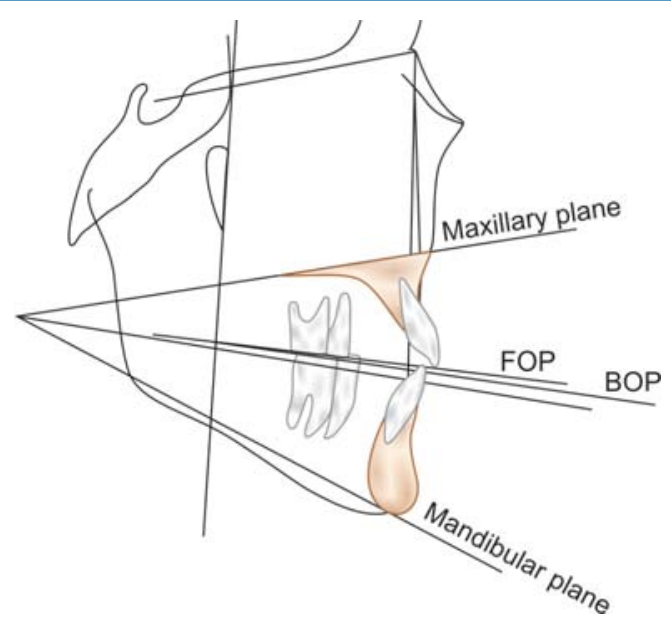

Fig. 3: Linear and angular measurement

Bm: Point B projected in perpendicular fashion onto the MM bisector.

$A b$ : Point A projected in perpendicular fashion onto the bisecting occlusal plane.

$\mathrm{Bb}$ : Point B projected in perpendicular fashion onto the bisecting occlusal plane.

\section{Angular Measurements}

With a large protractor measuring to $0.5^{\circ}$, the following angular measurements were made:

1. ANB angle and maxillomandibular bisector plane angle.

2. The angulations of FOP and BOP and the maxillo mandibular bisector plane angle to the $\mathrm{PM}$ vertical plane.

3. The angulations of FOP and BOP to the maxillo mandibular bisector plane angle.

\section{Linear Measurements}

With a steel ruler divided into $0.5 \mathrm{~mm}$, the relationship of point $A$ to point $B$ was measured to each occlusal plane and to maxillomandibular bisector plane angle with the Wits technique.

\section{RESULTS}

The angular and linear measurements between adults and children are shown in Table 1. The standard error of the study was done using double determination test for various cephalometric measures for males (Table 2) and females (Table 3). The errors ranged from 0.89 to 1.12 . There is less error when using bisecting occlusal plane and maxillomandibular bisector compared with FOP 0.89 to 0.74

\section{The Error in Linear Measurement}

All of the errors, except FOP W its measurement, are in normal range; these errors ranged from 0.30 to 0.85 . 
Maxillomandibular Plane Angle Bisector (MM) Adjunctive to Occlusal Plane

\begin{tabular}{|c|c|c|c|c|c|}
\hline \multicolumn{2}{|l|}{ Groups } & \multirow{2}{*}{$\frac{N}{30}$} & \multirow{2}{*}{$\begin{array}{c}\text { Mean } \\
83.067\end{array}$} & \multirow{2}{*}{$\frac{\text { Std. deviation }}{2.067}$} & \multirow{2}{*}{$\begin{array}{c}T \\
0.3020\end{array}$} \\
\hline SNA & Adults & & & & \\
\hline & Children & 30 & 82.833 & 3.687 & $p=0.763 \mathrm{NS}$ \\
\hline \multirow[t]{2}{*}{ SNB } & Adults & 30 & 77.433 & 9.460 & 0.4690 \\
\hline & Children & 30 & 76.567 & 3.598 & $p=0.641 \mathrm{NS}$ \\
\hline \multirow[t]{2}{*}{ ANB } & Adults & 30 & 2.433 & 1.960 & 6.5010 \\
\hline & Children & 30 & 6.467 & 2.776 & $\mathrm{p}=0.001 \mathrm{VHS}$ \\
\hline \multirow[t]{2}{*}{ MM } & Adults & 30 & 23.433 & 4.091 & 2.0900 \\
\hline & Children & 30 & 25.767 & 4.546 & $p=0.041 \mathrm{Sig}$ \\
\hline \multirow[t]{2}{*}{ MM-PM } & Adults & 30 & 95.033 & 3.023 & 2.4940 \\
\hline & Children & 30 & 98.067 & 5.936 & $p=0.015$ Sig \\
\hline \multirow[t]{2}{*}{ FOP-PM } & Adults & 30 & 91.9000 & 4.2292 & 1.7780 \\
\hline & Children & 30 & 93.8333 & 4.1943 & $p=0.081 \mathrm{NS}$ \\
\hline \multirow[t]{2}{*}{ BOP-PM } & Adults & 30 & 90.3000 & 3.2605 & 1.5390 \\
\hline & Children & 30 & 92.5667 & 7.3798 & $p=0.129 \mathrm{NS}$ \\
\hline \multirow[t]{2}{*}{ FOP-MM } & Adults & 30 & 6.5667 & 2.1284 & 7.3440 \\
\hline & Children & 30 & 2.8667 & 1.7564 & $\mathrm{p}=0.001 \mathrm{VHS}$ \\
\hline \multirow[t]{2}{*}{ BOP-MM } & Adults & 30 & 6.3333 & 2.0398 & 0.7840 \\
\hline & Children & 30 & 5.9333 & 1.9106 & $p=0.436 \mathrm{NS}$ \\
\hline \multirow[t]{2}{*}{$A B-F O P$} & Adults & 30 & $3.333 E-02$ & 1.9911 & 3.0350 \\
\hline & Children & 30 & 2.1333 & 3.1142 & $\mathrm{p}=0.004 \mathrm{VHS}$ \\
\hline \multirow[t]{2}{*}{$A B-B O P$} & Adults & 30 & 0.8333 & 2.1509 & 4.2450 \\
\hline & Children & 30 & 2.7333 & 4.0678 & $p=0.001$ VHS \\
\hline \multirow[t]{2}{*}{ AB-MM } & Adults & 30 & -4.0000 & 2.16556 & $7.2000 \mathrm{E}-02$ \\
\hline & Children & 30 & -3.96667 & 1.29943 & $p=0.943 \mathrm{NS}$ \\
\hline
\end{tabular}

NS: Nonsignificant; Sig: Significant; VHS: Very highly significant

\begin{tabular}{|c|c|c|c|c|c|}
\hline Group male & & $\mathrm{N}$ & Mean & Std. deviation & $\mathrm{T}$ \\
\hline \multirow[t]{2}{*}{ SNA } & Adults & 15 & 83.0667 & 2.4919 & 0.8710 \\
\hline & Children & 15 & 82.0000 & 4.0356 & $p=0.391 \mathrm{NS}$ \\
\hline \multirow[t]{2}{*}{ SNB } & Adults & 15 & 73.9333 & 12.4296 & 0.5130 \\
\hline & Children & 15 & 75.667 & 4.0649 & $p=0.612 \mathrm{NS}$ \\
\hline \multirow[t]{2}{*}{ ANB } & Adults & 15 & 2.7333 & 1.3870 & 3.7050 \\
\hline & Children & 15 & 6.333 & 3.4983 & $p=0.001 \mathrm{VHS}$ \\
\hline \multirow[t]{2}{*}{ MM } & Adults & 15 & 25.1333 & 3.9797 & 0.5810 \\
\hline & Children & 15 & 25.9333 & 3.5550 & $p=0.566 \mathrm{NS}$ \\
\hline \multirow[t]{2}{*}{ MM-PM } & Adults & 15 & 95.0667 & 3.6541 & 1.3860 \\
\hline & Children & 15 & 97.2000 & 4.7087 & $p=0.177 \mathrm{NS}$ \\
\hline \multirow[t]{2}{*}{ FOP-PM } & Adults & 15 & 92.4667 & 4.6270 & 1.3350 \\
\hline & Children & 15 & 94.4667 & 3.5024 & $p=0.193 \mathrm{NS}$ \\
\hline \multirow[t]{2}{*}{ BOP-PM } & Adults & 15 & 90.4000 & 3.542 & 0.4210 \\
\hline & Children & 15 & 91.267 & 7.136 & $p=0.677 \mathrm{NS}$ \\
\hline \multirow[t]{2}{*}{ FOP-MM } & Adults & 15 & 5.733 & 1.907 & 5.7250 \\
\hline & Children & 15 & 2.667 & 0.816 & $p=0.001 \mathrm{VHS}$ \\
\hline \multirow[t]{2}{*}{ BOP-MM } & Adults & 15 & 6.3333 & 1.175 & 0.3260 \\
\hline & Children & 15 & 6.1333 & 2.006 & $p=0.747 \mathrm{NS}$ \\
\hline \multirow[t]{2}{*}{$A B-F O P$} & Adults & 15 & 0.533 & 1.885 & 1.2200 \\
\hline & Children & 15 & 1.933 & 4.026 & $p=0.233 \mathrm{NS}$ \\
\hline \multirow[t]{2}{*}{$A B-B O P$} & Adults & 15 & $6.667 \mathrm{E}-02$ & 2.404 & 1.7960 \\
\hline & Children & 15 & 2.667 & 5.066 & $p=0.083 \mathrm{NS}$ \\
\hline \multirow[t]{2}{*}{$A B-M M$} & Adults & 15 & -4.26667 & 1.48645 & 0.23000 \\
\hline & Children & 15 & -4.13333 & 1.68466 & $p=0.84 \mathrm{NS}$ \\
\hline
\end{tabular}

NS: Nonsignificant; VHS: Very highly significant

\section{Comparison between Adults and Children}

\section{Angular Measurements}

1. ANB angle: The mean value for the adults (2.433) and standard deviation (1.960), for children the mean value is 6.467 and standard deviation (2.776); on statistical comparison they show very significant difference.

2. Maxillomandibular bisector: The mean value for adults (23.433) and standard deviation of (4.091), for children the mean value is (25.767) and standard 


\begin{tabular}{|c|c|c|c|c|c|}
\hline \multicolumn{2}{|c|}{ Group female } & \multirow{2}{*}{$\begin{array}{r}N \\
15\end{array}$} & \multirow{2}{*}{$\begin{array}{c}\text { Mean } \\
83.0667\end{array}$} & \multirow{2}{*}{$\begin{array}{c}\text { Std. deviation } \\
1.6242\end{array}$} & \multirow{2}{*}{$\begin{array}{l}T \\
0.6440\end{array}$} \\
\hline SNA & Adults & & & & \\
\hline & Children & 15 & 83.6667 & 3.2219 & $p=0.525 \mathrm{NS}$ \\
\hline \multirow[t]{2}{*}{ SNB } & Adults & 15 & 79.9350 & 3.1560 & 1.0580 \\
\hline & Children & 15 & 77.4667 & 3.2450 & $p=0.193 \mathrm{NS}$ \\
\hline \multirow[t]{2}{*}{ ANB } & Adults & 15 & 2.1333 & 2.4162 & 5.6060 \\
\hline & Children & 15 & 6.6000 & 1.9198 & $p=0.001 \mathrm{VHS}$ \\
\hline \multirow[t]{2}{*}{ MM } & Adults & 15 & 21.7333 & 3.5550 & 2.2900 \\
\hline & Children & 15 & 25.6000 & 5.4876 & $p=0.03 \mathrm{Sig}$ \\
\hline \multirow{2}{*}{ MM-PM } & Adults & 15 & 95.0000 & 2.3604 & 2.0580 \\
\hline & Children & 15 & 98.9333 & 7.0150 & $p=0.049 \mathrm{Sig}$ \\
\hline \multirow{2}{*}{ FOP-PM } & Adults & 15 & 91.3333 & 3.8668 & 1.1690 \\
\hline & Children & 15 & 93.2000 & 4.8285 & $p=0.252 \mathrm{NS}$ \\
\hline \multirow[t]{2}{*}{ BOP-PM } & Adults & 15 & 90.200 & 3.075 & 1.7260 \\
\hline & Children & 15 & 93.867 & 7.633 & $p=0.095 \mathrm{NS}$ \\
\hline \multirow[t]{2}{*}{ FOP-MM } & Adults & 15 & 7.400 & 2.063 & 5.3350 \\
\hline & Children & 15 & 3.067 & 2.374 & $p=0.001$ VHS \\
\hline \multirow[t]{2}{*}{ BOP-MM } & Adults & 15 & 6.333 & 2.690 & 0.7190 \\
\hline & Children & 15 & 5.733 & 1.792 & $p=0.478 \mathrm{NS}$ \\
\hline \multirow[t]{2}{*}{ AB-FOP } & Adults & 15 & 0.467 & 2.031 & 3.5440 \\
\hline & Children & 15 & 2.333 & 2.289 & $p=0.01$ VHS \\
\hline \multirow[t]{2}{*}{ AB-BOP } & Adults & 15 & -1.333 & 1.893 & 4.6250 \\
\hline & Children & 15 & 2.800 & 2.933 & $p=0.001 \mathrm{VHS}$ \\
\hline \multirow[t]{2}{*}{$A B-M M$} & Adults & 15 & -3.73333 & 2.71153 & $9.2000 \mathrm{E}-02$ \\
\hline & Children & 15 & -3.80000 & 0.77460 & $p=0.928 \mathrm{NS}$ \\
\hline
\end{tabular}

NS: Nonsignificant; Sig: Significant; VHS: Very highly significant

deviation (4.556); on comparison, they shows the significant difference.

3. FOP, BOP and maxillomandibular planes angle measured to PM vertical plane.

a. Maxillomandibular bisector to PM vertical: The mean value for adults is (95.033) and standard deviation (3.023). The mean value for children (98.067) and standard deviation (5.936).

b. Functional occlusal plane to PM vertical: The mean value for adults is (91.900) and standard deviation (4.2292) and mean value for children is (93.833) standard deviation is (4.1943).

c. Bisecting occlusal plane to PM vertical: The mean value for adults is (90.300) and standard deviation (3.2605) and the mean value for the children is (92.560) and standard deviation is (7.3798).

When compared all the three planes to PM vertical, there is definite change in cant of functional occlusal plane to PM vertical than compared to bisecting occlusal plane and maxillomandibular bisector but it is not very significant.

4. Bisecting occlusal plane and functional occlusal planes angle measured to maxillomandibular bisector.

a. Functional occlusal plane to maxillomandibular bisector: The mean value for the adults is (6.5667) in standard deviation (2.1284) the mean value for children is (2.8607) and standard deviation is (1.7564). b. Bisecting occlusal plane to maxillomandibular bisector: The mean value for adults is (6.333) and standard deviation is (2.0398) and the mean value of children is (5.933) standard deviation is (1.9106).

On comparison, there is significant difference between the FOP and maxillomandibular bisector than compared to BOP to maxillomandibular bisector.

\section{Linear Measurements}

a. A and B values to FOP: The mean value for adults is (3.333) and standard deviation is (1.9911), the mean value for children is (2.133) and standard deviation is (3.2242).

b. $A$ and $B$ value measured to $B O P$ : The mean value for adults is (0.8333) and standard deviation is (2.1509) and mean value for children is (2.7333) and standard deviation is (4.0075).

c. $A$ and $B$ value measured to maxillomandibular bisector: The mean value for the adults is (4.000), standard deviation is (2.1055) and children (3.9907), standard deviation (1.299); there is very significantal value of $A$ and $B$ to $F O P$ when compared $A$ and $B$ values to $B O P$ and maxillomandibular bisector.

\section{Comparison between Male Adults and Children}

\section{Angular Measurements}

The values which are statistically significant are A NB angle and FOP to maxillomandibular bisector. The other values are not significant. 


\section{Linear Measurements}

On comparison between male adults and children, none of values showed any significant variation.

\section{Comparison between Female Adults and Children}

\section{Angular Measurement}

The value which are statistically significant are A N B angle, maxillomandibular bisector angle and maxillomandibular bisector to PM vertical plane and FOP to maxillomandibular bisector angle.

\section{Linear Measurement}

On comparison between female adult and children. The A and $B$ to $F O P$ shows very significant variations than $A$ and $B$ values compared to $B O P$ and maxillomandibular bisector.

\section{DISCUSSION}

A $n$ accurate AP measurement of jaw relationships is critically important in orthodontic treatment planning. The most popular parameter for assessing the sagittal jaw relationship remains the ANB angle, but it can be affected by various factors and can be misleading. A popular alternative that the $W$ its appraisal does not depend on cranial landmarks or rotation of the jaws but still has problem of correctly identifying the functional occlusal plane, which can sometime be impossible. Furthermore, changes of $W$ its measurement throughout orthodontic treatment might al so reflect change in FOP, rather than pure sagittal changes of the relationship of the jaw. ${ }^{2,3}$

This study was undertaken to analyze the clinical usefulness of the maxillomandibular bisector, its reproducibility, its validity and its relationship to the FOP, the BOP and the nature of its cant.

The SNA, SNB and subsequently ANB angle does not accurately describe the sagittal maxillary and mandibular apical base relationship and this was attributed to normal variations in the spatial position both sella and nasion ${ }^{2}$ and this changes with age, ${ }^{6}$ the $W$ its analysis is an adjunct to the ANB angle and is not meant to be considered alone as defining the sagittal relationship. ${ }^{7-9}$

Vertical growth rotations are part of jaw development in growing children and thus measurement of anterior, posterior relationship, with the W its technique, should be made to a plane that rotates with jaws. ${ }^{10,11}$

In the present study, it was found that the maxillary mandibular plane angle did not show any significant differences when compared betw een children and adults and also between male and female as shown by Sherman et al. ${ }^{12}$
The presence study, shows slight increase in the angle between the maxillomandibular bisector and the functional occlusal plane which is statistically very highly significant, similar to that found by Jarvinen. ${ }^{14}$ This implies a change in the cant of the FOP and the W its value using FOP showing greater difference than the normal.

When compared using Wits appraisal, the BOP Wits value had a higher relationship to dental measures than the FOP. ${ }^{13,14}$ The rotation of the reference plane tends to mask the true value of linear changes between two distinct points when measurement is made to it during rotation. ${ }^{15,16}$

Lei Zhou et al $^{17}$ found that the anteroposterior relationship of the dental arch and jaw-base fail to match in atleast one out of every three individuals and that linear measurement of anteroposterior jaw-base relationships is a more valid reflection of the dental arch relationship than angular measurements.

In the present study, the Wits value measured to BOP the showed a class II tendency in the children and it increased with the age (becomes more positive) which suggests a slight increase in the maxillary base and decrease in mandibular base than compared to adults. The maxillomandibular bisector angle measured to PM vertical showed that angle moved downward and backward with the age which reflects the direction and the amount of total growth rotation of the dental alveolar complex.

Point $A$ and $B$ move the same amount and direction as their dental basis and so their true anterior-posterior relationship was effectively measured when using this plane. Since, the maxillomandibular bisector lies beneath the functional occlusal plane, therefore, B is projected ahead of point $A$ for normal occlusion, resulting in a negative value. In females, children showed slightly increased mean value, i.e. $(-3.800)$ than compared to adults $(-3.733)$, whereas in males, adults showed slightly increased value $(-4.266)$ than children $(-4.133)$.

\section{CONCLUSION}

Following conclusions were drawn from the present study:

1. The maxillomandibular bisector is a more reproducible reference plane compared with BOP or FOP.

2. The maxillomandibular bisector for reference plane shows slight change, when compared with BOP and FOP but the $W$ its value measured shows nothing significant when compare to FOP and BOP.

3. Lying at an angle and inferior to the other occlusal planes, measurement to the maxillomandibular bisector is not obscured by the outline of the teeth. However, readings to it are of a negative value for skel etal because of downward cant of the planes interiorly. 
4. M ean values for the Wits assessment made to maxillomandibular bisector is adults male more (4.2666) than adults female - 3.7333 and children male (4.1333); children female (3.800).

5. The anterioposterior measurement made in conjunction with as skull base reference, such as the ANB angle.

W e do not suggest that clinicians should totally disregard any previously established cephalometric measurements rather, maxillomandibular bisector angle plane, enriches the current cephalometric tools availability to the clinician and enables better diagnosis and treatment planning for patient.

Since, this study was conducted using lateral cephalograms which provides only a two-dimensional vision of the morphological features, a study that can permit threedimensional vision like three-dimensional CT reconstruction provided a better understanding of features.

\section{CLINICAL SIGNIFICANCE}

The maxillomandibular plane angle bisector was found to be a more reproducible and valid reference plane, than the FOP and BOP. M axillomandibular planes angle bisector may be a useful adjunct for the cephalometric assessment of sagittal relationship of the patient.

\section{REFERENCES}

1. Broadent BH. A new X-ray technique and its application to orthodontics. A ngle Orthod 1931;1:45-66.

2. Jacobson A. The W its appraisal of jaw disharmony. A m J Orthod and Dentofac Orthod 2003;124:470-79.

3. A ranha CA, Galvao N, M adeira M C. Comparative study between W its appraisal and I-line. A $\mathrm{m} J$ Orthod and Dentofac Orthop 1985;55:181-89.

4. Jenkins DH. A nalysis or orthodontic deformity employing lateral cephalometric radiography. A m J Orthod and Dentofac Orthop 1955;41:442-52.

5. Harvold EP. Some biologic aspects of orthodontic treatment in the transitional dentition. A m J Orthod and Dentofac Orthop 1963;49:1-14.

6. Bishara SE, Fahl JA, Peterson L C. L ongitudinal changes in the ANB angle and Wits appraisal: Clinical implications. Am J Orthod and Dentofac Orthop 1983;84:133-39.

7. Jacobson A. Update on the 'W its' appraisal. A ngle Orthod 1988;58:205-19.

8. Chan M D. An evaluation of the 'W its' appraisal using pre and post-treatment cephalometric values san Diego: U niversity of Southern California School of Dentistry, Thesis 1985.
9. Rotberg S, Fried N, Kane J, Shapiro E. Predicting the 'W its' appraisal from the ANB angle. Am J Orthod and Dentofac Orthop 1980;77:636-42.

10. Roth R. The Wits appraisal - its skeletal and dentoalveolar background. Eur J Orthod 1982;4:21-28.

11. Rushton $A$, Cohen $A M, L$ inney $A D$. The relationship and reproducibility of angle ANB and the W its appraisal. B rJ Orthod 1991;18:225-31.

12. Sherman SL, W oods M, N anda RS. The longitudinal effects of growth on the W its appraisal. A m J Orthod and D entofac O rthop 1988; $93: 429-36$.

13. Foley TF, et al. The reliability of three sagittal reference planes in the assessment of the class II treatment. Am J Orthod and Dentofac Orthop 1997;112:320-29.

14. J arvinen S. A nalysis of ANB angle a statistical appraisal. A m J Orthod and Dentofac Orthop 1985;87:144-46.

15. Schudy FF. The rotation of the mandible resulting from growth: Its implications in orthodontic treatment. Angle Orthod 1965:35:36-50

16. Creekmore TD. Inhibition or stimulation of vertical growth of the facial complex. Angle Orthod 1967;37:285-97.

17. Lei Zhou, Chung-Wai Mok, Urban Hagg, Colman M cGrath, $M$ argareta B endeus, J ohn W u. A nteroposterior dental arch and jaw-base relationships in a population sample. Angle Orthod 2008;78:1023-29.

\section{ABOUT THE AUTHORS}

\section{Chanamallappa R Ganiger (Corresponding Author)}

Reader, Department of Orthodontics and Dentofacial Orthopedics School of Dental Sciences, K rishna Institute of M edical Sciences Deemed University, Karad, Satara, M aharashtra, India, Phone: 09823463681, e-mail: channapparganiger@gmail.com

\section{US Krishna Nayak}

Dean A cademics, Senior Professor and Head, Department of Orthodontics and Dentofacial Orthopedics, AB Shetty Memorial Institute of D ental Sciences, M angalore, K arnataka, India

\section{KU Cariappa}

Senior Professor, Department of Orthodontics and Dentofacial Orthopedics, AB Shetty Memorial Institute of Dental Sciences $M$ angalore, K arnataka, India

\section{AR Yusuf Ahammed}

Senior Lecturer, Department of Orthodontics and Dentofacial Orthopedics, School of Dental Sciences, KIMSDU, Satara Maharashtra, India 